\title{
Olfactory Impairments in Patients with Unilateral Cerebellar Lesions Are Selective to Inputs from the Contralesional Nostril
}

\author{
Joel D. Mainland, ${ }^{1}$ Bradley N. Johnson, ${ }^{2}$ Rehan Khan, ${ }^{1}$ Richard B. Ivry, ${ }^{3}$ and Noam Sobel ${ }^{1,2,3}$ \\ ${ }^{1}$ Helen Wills Neuroscience Institute, ${ }^{2}$ Program in Bioengineering, and ${ }^{3}$ Department of Psychology, University of California at Berkeley, Berkeley, California \\ 94720
}

\begin{abstract}
Functional imaging studies of olfaction have consistently reported odorant-induced activation of the cerebellum. However, the cerebellar role in olfaction remains unknown. We examined the olfactory and olfactomotor abilities of patients with unilateral cerebellar lesions, comparing performance within subjects across nostrils, as well as between subjects with age-matched and young controls. Regarding olfactory performance, initial testing revealed that patients had a contralesional impairment in olfactory identification but not olfactory detection threshold. However, when tested under conditions that prevented compensatory sniffing strategies, the patients also exhibited a contralesional olfactory detection impairment. Regarding olfactomotor function, a healthy olfactomotor system generates sniffs that are (1) sufficiently vigorous and (2) inversely proportional to odorant concentration in sniff mean airflow velocity, maximum airflow velocity, volume, and duration. Patients' sniffs were lower in overall airflow velocity and volume in comparison with control participants. Furthermore, reduced sniff velocity predicted poorer detection thresholds in patients. Finally, whereas young controls used concentration-dependent sniffs, there was a trend in that direction only for age-matched controls. Patients used sniffs that were concentration invariant. In conclusion, cerebellar lesions impacted olfactory and olfactomotor performance. These findings strongly implicate an olfactocerebellar pathway prominent in odor identification and detection that functionally connects each nostril primarily to the contralateral cerebellum.
\end{abstract}

Key words: sensorimotor; olfactory; cerebellum; odor; sniff; intensity

\section{Introduction}

Although the cerebellum is not traditionally considered part of the olfactory system, cerebellar activity has been consistently observed in functional imaging studies of olfaction (Small et al., 1997; Yousem et al., 1997; Sobel et al., 1998; Qureshy et al., 2000; Savic et al., 2000; Zatorre et al., 2000; Cerf-Ducastel and Murphy, 2001; Ferdon and Murphy, 2003). Despite this, the pathways through which olfactory information reaches the cerebellum and the functional role of the cerebellum in olfaction remain unknown.

To probe cerebellar involvement in olfaction, we measured olfactory performance in patients with focal unilateral cerebellar lesions. Two recent reports suggested that patients with bilateral cerebellar degeneration exhibit a significant decrement in olfactory identification (Abele et al., 2003; Connelly et al., 2003) that was persistent despite normal olfactory detection thresholds

Received Sept. 5, 2004; revised May 16, 2005; accepted May 25, 2005

This work was supported by National Science Foundation (NSF) Grant BCS-0345922 and National Institutes of Health Grants NS 30256 and NS 40813. J.D.M. was supported by an NSF Graduate Research Fellowship. We thank Arak Elite.

Correspondence should be addressed to either Dr. Joel D. Mainland or Dr. Noam Sobel, 3210 Tolman Hall, MC1650, University of California at Berkeley, Berkeley, CA 94720. E-mail: mainland@uclink.berkeley.edu or nsobel@socrates.berkeley.edu.

DOI:10.1523/JNEUROSCI.0920-05.2005

Copyright $\odot 2005$ Society for Neuroscience $\quad$ 0270-6474/05/256362-10\$15.00/0
(Abele et al., 2003). This profile of impaired identification despite intact thresholds raises the possibility that cerebellar lesions may impair cognitive mechanisms related to object identification in general, rather than olfactory identification per se. Alternatively, patients' normal detection performance may have resulted from a compensatory strategy. For example, they may have taken longer sniffs to acquire more olfactory information, similar to what healthy subjects do under conditions of limited nasal airflow (Sobel et al., 2000). Considering the hierarchical nature of olfactory processing (Savic et al., 2000), such compensatory behavior may have been sufficient to obscure impairments in olfactory detection, but not identification. To address the hypothesis that focal cerebellar lesions impair olfaction, we evaluated patients' olfactory detection and identification performance against published norms and in comparison with young and agematched healthy control subjects. To address the hypothesis that compensatory sniffing obscured part of this impairment, we tested patients and controls under conditions that prevented compensatory sniff behavior.

We also ask whether monorhinal performance in unilateral focal lesion patients can shed light on the pathway that links the nose to the cerebellum. Unilateral cerebellar lesions lead to asymmetric impairments in motor function and vision (Snider and Stowell, 1944; Suzuki and Keller, 1988; Bjaalie and Brodal, 1989; Hore et al., 1991). Thus, we hypothesized that comparing perfor- 


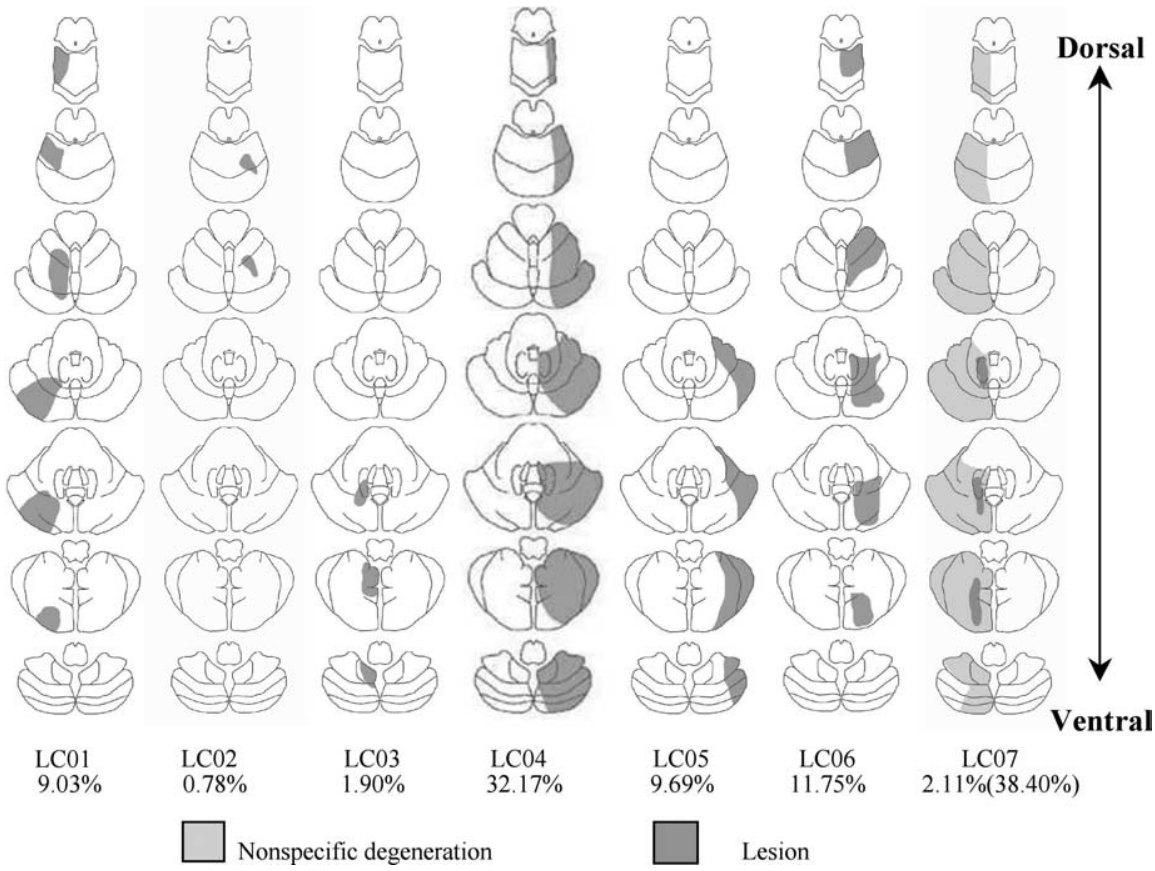

Figure 1. Cerebellar lesions in the seven patients. MRI scans were analyzed by a neurologist, and the extent of pathology was sketched on seven axial cerebellar slices. Four lesions resulted from damage associated with stroke (LC01, LC02, LC04, LC06), and three lesions resulted from damage associated with tumor resection ( $\mathrm{LCO3}, \mathrm{LC05}, \mathrm{LCO7})$. Below each template is the percentage of cerebellum lesioned. In patient LCO7, the first value relates to lesion damage, and the second value relates to nonspecific degeneration.

mance between conditions in which odorants were restricted to one nostril or the other would enable us to determine whether the link from nose to cerebellum is ipsilateral, contralateral, or bilateral.

Finally, we explore the hypothesis that the cerebellum may control the olfactomotor system (Sobel et al., 1998; Johnson et al., 2003). The olfactomotor system generates sniffs that are (1) sufficiently vigorous to sample the stimulus and (2) inversely proportional to odorant concentration (Laing, 1983; Johnson et al., 2003). We predicted that cerebellar lesions might impair either of these olfactomotor components. Specifically, patients may employ sniffs that are either insufficiently vigorous and/or unrelated to odorant concentration, consistent with the idea that the cerebellum modulates motor systems to optimize sampling of sensory information (Bower, 1997a,b).

\section{Materials and Methods \\ Subjects}

Experimental procedures were approved by the UC Berkeley Committee for The Protection of Human Subjects, and informed written consent was obtained from each subject.

Patients. Seven male patients with focal unilateral cerebellar lesions resulting from stroke (four patients) or tumor resection (three patients) participated in the study. Their mean age was 57 years (range, 45-76 years), and they averaged 14.3 years of education (range, 11-18 years). Six of the seven patients scored normally on the Mini-Mental State Examination (range, 28-30), and one had a score of 25. All of the patients were able to name pictured objects on the Boston Naming Test (BNT) short form within the normal range (mean items correct of 15, $14.17 \pm$ 1.17; range, 12-15). Furthermore, the patients scored significantly above average on the Wechsler Memory Scale (WMS) digit span task (scaled score, $11.83 \pm 0.60 ; t_{(5)}=3.05 ; p<0.03$; range, $10-14$ ) and were not significantly different from normal scores on any of the 13 subtests of the Wechsler Adult Intelligence Scale (WAIS-III) except symbol search (mean scaled score, $8.00 \pm 0.76 ; t_{(6)}=2.646$; $p<0.04$ ) and letter-number sequencing (mean scaled score, $\left.6.4 \pm 1.1 ; t_{(4)}=3.207 ; p<0.03\right)$.

None of the patients had a history of broken nose or surgery of the nasal passage. Three patients reported being active smokers, averaging 10 cigarettes per day. Two patients reported never having smoked, and the remaining two patients reported having not smoked for at least 20 years.

Reconstructions of the lesions, based on magnetic resonance imaging (MRI) scans, are plotted in Figure 1. Lesions were restricted to the left side of the cerebellum in three of the patients and to the right side in the other four. None of the patients had extracerebellar lesions. The average time since lesion onset was 6.4 years, with a minimum of 5 years.

Healthy subjects. Two groups of healthy subjects were tested on all tasks. The first group consisted of seven subjects matched for age (mean, 58 years; range, 46-75 years) and gender (seven male). Two of these subjects were current smokers, and one had been a smoker previously but not for the past 40 years.

The second group consisted of seven college students (three male), with a mean age of 24 years (range, 20-30 years). One had been a smoker previously, but not for the past 3 months.

Each patient was assigned one control subject based on age, and one college student by random selection for analysis. Members of the matched pair were tested with the same item and nostril order; thus, if the patient's right nostril was contralesional, the healthy subject's right nostril was designated "contralesional."

\section{Tasks}

Olfactory identification. We assessed general olfactory ability using a standardized test [University of Pennsylvania Smell Identification Test (UPSIT)] (Doty et al., 1984). The UPSIT consists of 40 four-alternative forced-choice items. Each test item consists of 10-50 $\mu \mathrm{m}$ urea-formaldehyde polymer microencapsules fixed in a proprietary binder and positioned on a brown strip at the bottom of the test booklet. The experimenter released the stimulus by scratching the strip with a pencil tip in a standardized manner. A multiple-choice question with four alternative responses is provided with each odorant strip. For example, one item reads as follows: "This odor smells most like a) chocolate; b) banana; c) onion; d) fruit punch." The subject was allowed to read the items, and the experimenter also read the items aloud. The subjects were given $30 \mathrm{~s}$ to answer after odorant delivery. A $30 \mathrm{~s}$ intertrial interval separated trials to minimize carryover effects.

During testing, we occluded one nostril with odorless Microfoam tape (3M Corporation, St. Paul, MN) cut to fit over the borders of the naris. Participants were tested on 20 of the UPSIT items with one nostril and the remaining 20 items with the other nostril. A number of studies have validated this unilateral method of testing (Doty, 1992; Good et al., 2003). Order and items were counterbalanced across subjects and the order of test items was identical for a patient and his matched healthy participant. We scored the UPSIT by number correct of 20, corrected for smoking and years of education according to Good et al. (2003).

Auditory identification. To ask whether the profile of olfactory identification was specific to olfaction, we designed an auditory analog to the UPSIT. This test consisted of 40 four-alternative forced-choice items. Items were chosen from a set of everyday, nonverbal, digitized sounds (Marcell et al., 2000). Each sound was presented once through headphones to either the left or right ear. Simultaneously, a multiple-choice question with four alternative responses was presented to the subject on a monitor (e.g." "This sounds most like a) sawing; b) woodpecker; c) rain; 
d) telephone.") The subject was allowed to read the item, and the experimenter also read the items aloud, followed by a $30 \mathrm{~s}$ interval for the response. Similar to the UPSIT, we scored the auditory identification task by number correct of 20 . Testing of the auditory control was limited to five of the seven patients, because one patient was unavailable for the control task and a second patient was deaf in the right ear.

Olfactory detection thresholds. Detection thresholds were measured for the odorants propionic acid (PA) ( $\geq 99 \%$; Sigma, St. Louis, MO) and phenethyl alcohol (PEA) ( $\geq 99 \%$; Sigma). We chose these odorants because PA stimulates both the olfactory and trigeminal nerves whereas PEA stimulates only the olfactory nerve (Doty, 1995). Furthermore, PA has been the odorant of choice in experiments measuring odordependent sniffing (Kendal-Reed et al., 1998, 2001; Sobel et al., 2001; Walker et al., 2001; Johnson et al., 2003). Threshold tests were administered using two binary-dilution 20-step staircases, with the highest concentration set at $10 \% \mathrm{v} / \mathrm{v}$ phenylethyl alcohol in mineral oil and $0.1 \%$ propionic acid, respectively.

Olfactory thresholds for the two tested odorants were determined for each nostril using a maximum-likelihood adaptive staircase procedure modeled after Linschoten et al. (2001). The participants were blindfolded for this task. On each trial, two glass jars were presented in succession. One jar contained a given concentration of the odorant dissolved in mineral oil; the other jar contained mineral oil alone. The subject was asked to choose the jar with the strongest odor and, if undecided, required to guess (forced choice). The first trial commenced at step 1 (lowest concentration), and the staircase was terminated when the confidence interval fell below 0.5 log concentration steps in both directions or after 20 trials (because of adaptation and attention effects), whichever came first. One patient was unavailable for threshold testing in this condition.

Fixed-sniff detection threshold. To estimate the influence of possible compensatory sniffing strategies in patients, detection thresholds for PEA were measured a second time using a fixed-sniff method. Subjects wore a nasal mask with an attached spirometer to measure real-time nasal airflow. This flow rate was displayed as a continuous visual contour that could be compared by the subject with a target contour superimposed on the screen. The target contour was a square wave with a $1.5 \mathrm{~s}$ duration. Subjects were asked to match their sniff to the template and performed 10 practice trials. After the practice session, a threshold test was administered as above to each nostril. During the threshold test, subjects were instructed to focus on matching their sniff to the template during the threshold test as their primary task, thus generating a fixed sniff across nostrils. Subjects were first tested on their low-flow-rate nostril, and the gain was adjusted to allow them to easily match the template sniff. The gain was then kept at that setting for both nostrils.

Olfactomotor function. We assessed patients' olfactomotor function by measuring the effect of odorant concentration on four sniff airflow parameters: mean airflow velocity, maximum airflow velocity, sniff volume, and sniff duration. Odorants were delivered using an air-dilution olfactometer (Johnson et al., 2003). In brief, the system enabled switching from a no-odorant condition to an odorant condition in $<2 \mathrm{~ms}$. There were no auditory, visual, tactile, or thermal cues signaling the alteration between the two conditions. Subjects were fitted with a nasal mask attached to the olfactometer. The subject's sniff was monitored in real-time via a high-resolution pneumotachograph (high-sensitivity flow meter model 4719; Hans Rudolph, Kansas City, MO) coupled to a spirometer (ADInstruments, Grand Junction, $\mathrm{CO}$ ) attached to the nasal mask. The signal was amplified and digitally recorded at $100 \mathrm{~Hz}$. Sniffs were preprocessed by removing baseline offsets and aligned in time by setting the point at which the sniff passed from the expiratory phase to the inspiratory phase as time 0 . The offset of the sniff was set to the point at which the sniff returned to zero flow. Sniff volume was calculated by the trapezoidal Reimann sum method. Because of signal noise at the tail of the sniff, sniffs were truncated at $3 \mathrm{~s}$ for calculations of maximum flow, mean flow, and volume. Sniffs were not truncated for calculations of duration.

Subjects were trained to breath in and out of their mouth only, until an auditory instruction was presented. The instruction was as follows: "Please sniff after the tone." The olfactometer then delivered the odorant to the subject. After the sniff, subjects were required to rate odorant intensity on a scale using integers from 1 (no odor) to 9 (very strong) by entering their response on a keyboard. A $35 \mathrm{~s}$ intertrial interval preceded the onset of the next trial. During the delay, a trivia question followed by its answer was presented on the monitor to maintain the subject's attention.

Each block consisted of 17 trials. This number of trials was chosen as a compromise between the increased statistical power offered by a greater number of trials, and habituation effects that emerge with increased exposure to controlled olfactory events (Cain and Johnson, 1978). Each of the four concentration levels (clean air, low, medium, and high) was presented four times within a block of trials. The concentrations were presented pseudorandomly, counterbalanced for one-back trial history. The 16 test trials were always preceded by a clean air trial. Because this trial was not one-back counterbalanced, it was not included in the analyses.

In the first test session, subjects completed one monorhinal block with each nostril, as well as one block birhinally, counterbalanced for order across subjects. The odorants used were four concentrations of PA that corresponded to 0 (clean air), 9, 12, and $27 \mathrm{ppm}$. In the second testing session, we changed this protocol in two ways. First, because both patients and age-matched healthy subjects did not rate the medium concentration as significantly different in intensity from the low concentration, we substituted the $12 \mathrm{ppm}$ with $18 \mathrm{ppm}$ for a second testing session (for rationale and details, see Results). Second, to examine the possibility that odorant was reaching the epithelium before the sniff, the subject's real-time respiratory trace was used to trigger odorant delivery into the mask. Subjects were trained to breathe in and out of their mouths only, until an auditory instruction was presented. The instruction was as follows: "At the tone, sniff out and then in." The olfactometer then triggered the odorant by detecting outward expiration in the real-time respiratory trace. In other words, when the subject sniffed out, the odorant was delivered, and by the time the subject sniffed in, the odorant was present in the mask. Subjects then completed two blocks monorhinally with each nostril, as well as two blocks birhinally, counterbalanced for order across subjects. These modifications were conducted for both patients and controls; thus, there were no differences in testing procedures across groups. Furthermore, there were no differences in olfactomotor results between these two methods of odorant delivery timing, and the patients had no difficulty with either set of instructions. This was evident in the small number of faulty sniffs (sniffs that failed to trigger the olfactometer) taken throughout this study. Specifically, the number of faulty sniffs for the young healthy subjects, age-matched healthy subjects, and patients was $0.84 \%$ ( 6 of 714 ), $2.5 \%$ ( 18 of 714 ), and $2.24 \%$ (15 of 714 ), respectively. Data from these sniffs were eliminated from the analysis. Finally, as done previously by Johnson et al. (2003), we compared sniffs of the high-concentration odorant with sniffs of the low-concentration odorant. Such comparisons of concentration-dependent sniffing are sufficiently sensitive for clinical diagnosis of olfactory impairments (Frank et al., 2003).

Planned comparisons. Based on previous results (Abele et al., 2003), we predicted that patients would perform worse than control subjects on the olfactory identification and olfactomotor tasks, but not on olfactory detection. Furthermore, considering that patients had unilateral lesions, we predicted olfactory performance would be impaired in one nostril more than in the other. Although symptoms related to unilateral cerebellar lesions are more pronounced in ipsilateral movements, we did not make an a priori prediction concerning the affected side, given how little is known about the olfactocerebellar pathway.

\section{Results}

\section{Olfactory identification}

Normative data have been collected for the monorhinally administered modification of the UPSIT, a widely used, well normed and validated, standardized, and commercially available measure of olfactory identification (Good et al., 2003). All comparisons used the raw number of correct items of 20 on the UPSIT, cor- 
rected by years of education and smoking according to Good et al. (2003).

Planned contrasts revealed that patients scored significantly lower than both age-matched (mean patient, $15.36 \pm 0.61$; mean age-matched, $\left.16.14 \pm 0.50 ; F_{(1,18)}=4.58 ; p=0.046\right)$ and young controls (mean young control, $17.43 \pm 0.36 ; F_{(1,18)}=31.80 ; p<$ $0.0001)$. In patients, the contralesional nostril score was significantly lower than the ipsilesional nostril score (mean contralesional, $14.43 \pm 1.02$; mean ipsilesional, $16.29 \pm 0.52 ; F_{(1,18)}=$ $6.39 ; p<0.021)$. In contrast, there was no difference between nostrils in age-matched or young controls (mean age-matched contralesional, $16.28 \pm 0.52$; mean age-matched ipsilesional, $16.00 \pm 0.90 ; F_{(1,18)}=0.15 ; p=0.70 ;$ mean young contralesional, $17.71 \pm 0.42$; mean young ipsilesional, $17.14 \pm 0.59 ; F_{(1,18)}=$ $0.61 ; p=0.45)$. Thus, identification in the patient's contralesional nostril was poorer than identification in any single nostril of age-matched and young controls $\left(F_{(1,18)}=4.58 ; p<0.046\right)$.

\section{Auditory identification}

In addition to testing olfaction, the UPSIT requires the subject to recall previously experienced odors. The cerebellum has been implicated in memory storage and retrieval (Buckner et al., 1995; Moscovitch et al., 1995; Desmond and Fiez, 1998). To address the possibility that a generalized, multisensory memory impairment may underlie the lateralized deficits in the UPSIT test, we conducted an auditory identification control task.

Planned contrasts revealed that patients were impaired in comparison with both age-matched controls (mean patients, $15.8 \pm 0.98$; mean age-matched, $17.6 \pm 0.70 ; F_{(1,18)}=20.28 ; p<$ 0.00028 ) and young controls (mean, $18.4 \pm 0.45 ; F_{(1,18)}=16.74$; $p<0.00069)$. Critically, however, unlike the impairment in olfactory identification, this impairment was not lateralized (mean patient ipsilesional, $15.00 \pm 1.92$; mean patient contralesional, $16.6 \pm 0.51 ; F_{(1,18)}=2.36 ; p=0.14$; mean age-matched ipsilesional, $18.14 \pm 0.51$; mean age-matched contralesional, $18.14 \pm$ $0.34 ; F_{(1,18)}=0 ; p=1.00$; mean young ipsilesional, $18.28 \pm 0.78$; mean young contralesional, $17.57 \pm 0.61 ; F_{(1,18)}=0.47 ; p=$ $0.50)$.

\section{Olfactory detection}

PA threshold

A nested-design repeated-measures ANOVA with factors of subject nested within group (patient, age-matched healthy, young healthy) and side (ipsilateral matched, contralateral matched) failed to reveal significant effects (Fig. 2B).

\section{PEA threshold}

A nested-design repeated-measures ANOVA with factors of subject nested within group (patient, age-matched healthy, young healthy) and side (ipsilateral matched, contralateral matched) failed to reveal significant effects (Fig. 2C). In addition, the thresholds for all three groups in this condition were similar to previously published values for healthy subjects tested with PEA in mineral oil ( $-4.7 \mathrm{log}$ concentration) (Tsukatani et al., 2003).

\section{Fixed-sniff detection threshold}

Considering the lateralized contralesional impairment in olfactory identification, we found the symmetric olfactory detection threshold across nostrils to be puzzling. One possibility is that the patients were able to compensate for a detection impairment by taking longer or stronger sniffs. Given that we did not control sniffing in the initial detection experiment, we retested detection threshold of PEA while requiring the participants to use a constant sniff (Fig. 3A).

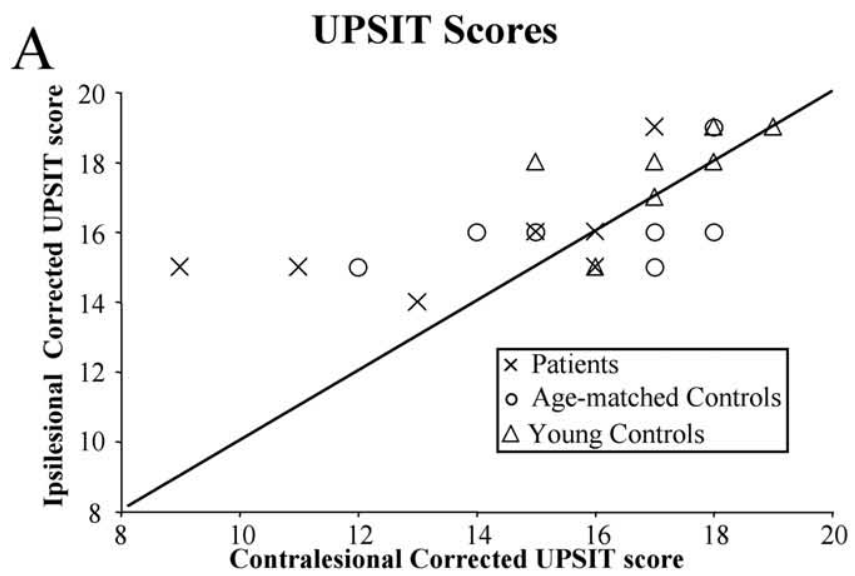

B

PA Thresholds

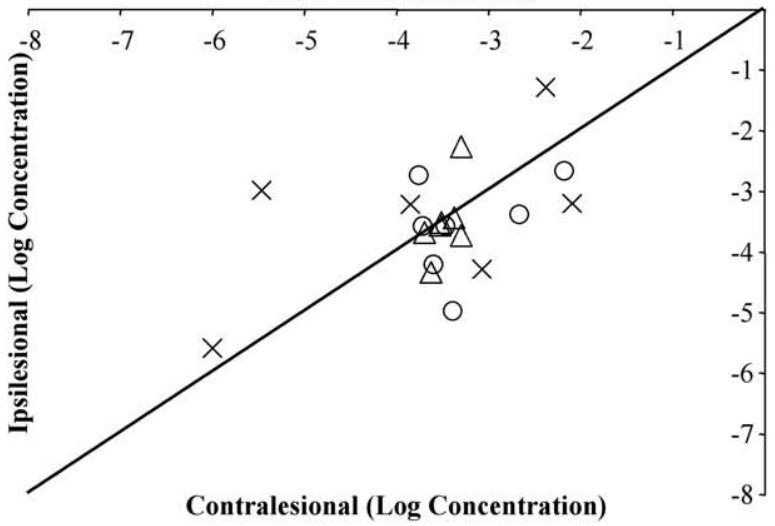

$\mathcal{C}$

PEA Thresholds

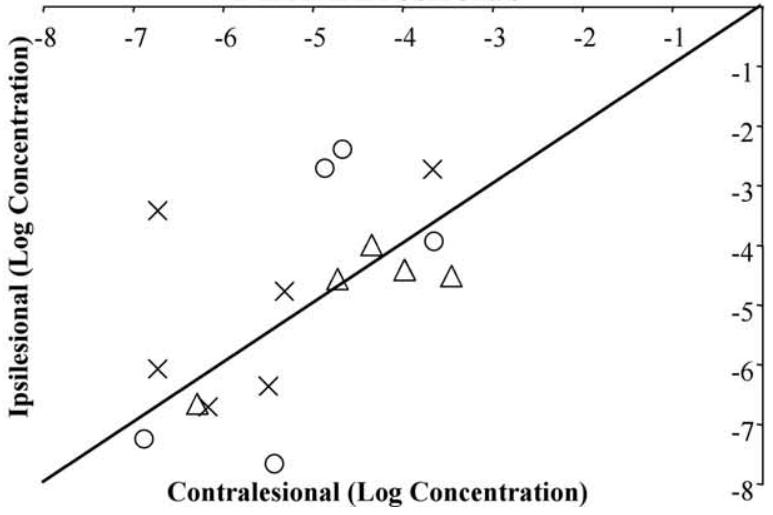

Figure 2. Performance on the odor identification and odor detection tasks. In each panel, the symbols represent results from one participant, either in the patient $(X)$, age-matched control $(\bigcirc)$, or young control $(\triangle)$ group. The diagonal line is the unit slope line. Points distributed uniformly around this line would correspond to identical performance with the left and right nostrils; points above the line correspond to the situation in which performance was better with the ipsilesional nostril. $\boldsymbol{A}$, Olfactory identification (UPSIT) raw, corrected scores. Higher numbers indicate better performance. Note that, although points representing the patients fell mostly above the line, both control groups were evenly distributed around the line. $B, C$, Detection thresholds for PA $(\boldsymbol{B})$ and PEA $(\boldsymbol{C})$. More negative values indicate better performance. All three groups were distributed around the line.

An ANOVA revealed a group effect $\left(F_{(2,18)}=41.64 ; p<\right.$ $0.0001)$. Planned contrasts showed that patients' thresholds were higher (worse) than age-matched controls (mean patient, $-1.40 \pm 0.66$; mean age-matched, $-4.55 \pm 0.49 ; F_{(1,18)}=$ $112.27 ; p<0.0001$ ) and young controls (mean young, $-4.87 \pm$ 


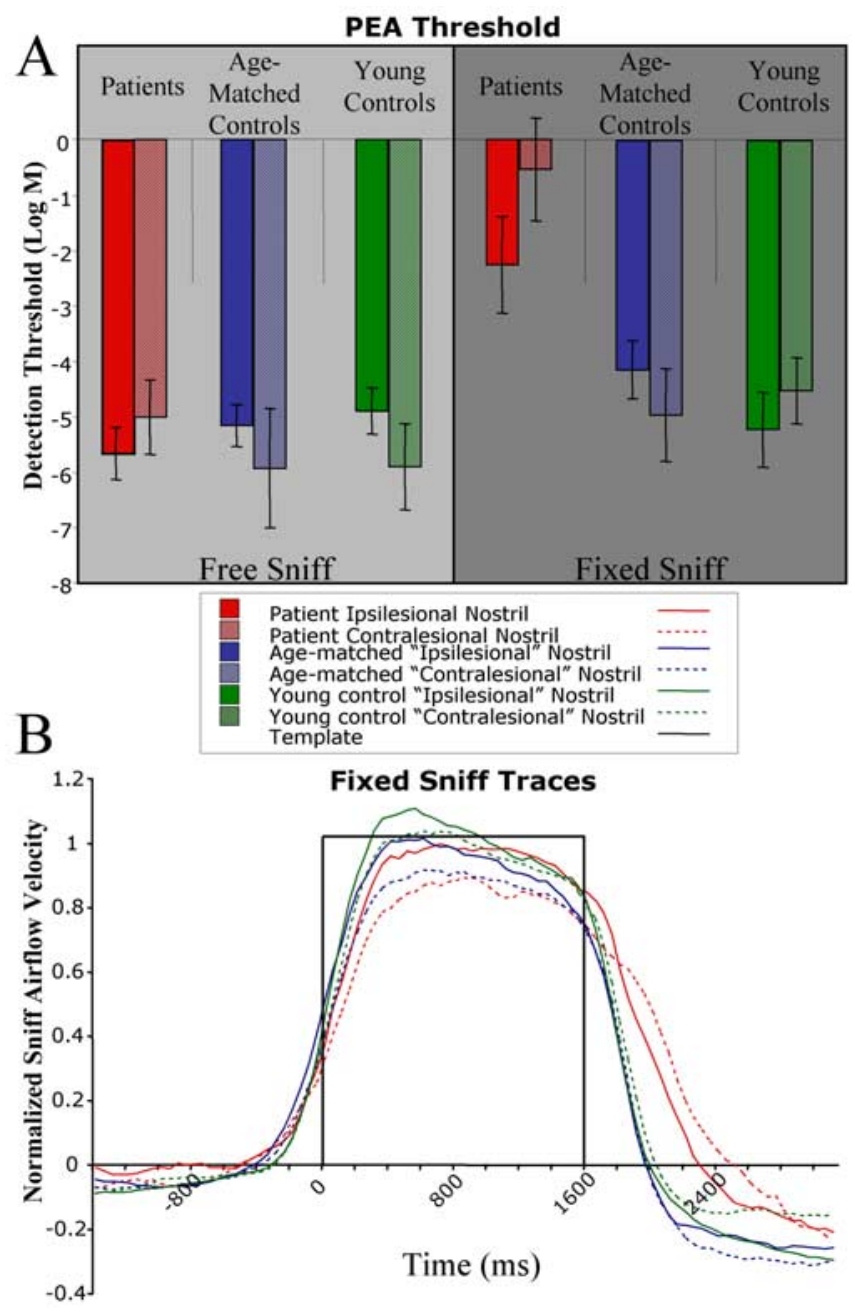

Figure 3. Performance on the fixed-sniff PEA threshold task. A, Average thresholds for the group are plotted. Error bars are SE. More negative values indicate better performance. Note that, in the forced-sniff task, patients' overall thresholds were much worse relative to controls, and that the impairment in threshold was lateralized, with the contralesional nostril showing a larger impairment. $\boldsymbol{B}$, Average sniff airflow produced by the subjects during the forced-sniff threshold task. Subjects were asked to match their respiratory trace to a square-wave template, shown in black. The task led subjects to take equal-magnitude sniffs across nostrils.

$\left.0.45 ; F_{(1,18)}=136.37 ; p<0.0001\right)$. Furthermore, patients' thresholds were worse in the contralesional than ipsilesional nostril (mean ipsilesional, $-2.26 \pm 0.87$; mean contralesional, $-0.54 \pm$ $\left.0.93 ; F_{(1,18)}=8.41 ; p<0.0096\right)$, but there were no lateralized effects in the age-matched controls (mean ipsilesional, $-4.78 \pm$ 0.89 ; mean contralesional, $\left.-4.31 \pm 0.48 ; F_{(1,18)}=0.62 ; p=0.44\right)$ or young controls (mean ipsilesional, $-4.73 \pm 0.69$; mean contralesional, $\left.-5.01 \pm 0.61 ; F_{(1,18)}=0.23 ; p=0.64\right)$.

Strikingly, a regression analysis showed that, across subjects, larger sniffs predicted lower (better) thresholds $\left(r=0.64 ; F_{(1,40)}\right.$ $=27.27 ; p<0.0001)$ (Fig. 4). This was true for patients $(r=0.60$; $\left.F_{(1,12)}=6.84 ; p<0.023\right)$ but not age-matched controls $(r=0.08$; $\left.F_{(1,12)}=0.078 ; p=0.78\right)$ or young controls $\left(r=0.511 ; F_{(1,12)}=\right.$ $4.24 ; p=0.062)$. Given that patients had slightly larger sniffs in the ipsilesional nostril compared with the contralesional nostril (Fig. $3 B$ ), it is possible that this small difference explains the asymmetry in detection threshold. However, the difference between the ipsilesional and contralesional mean velocity did not correlate with the difference between the ipsilesional and con-

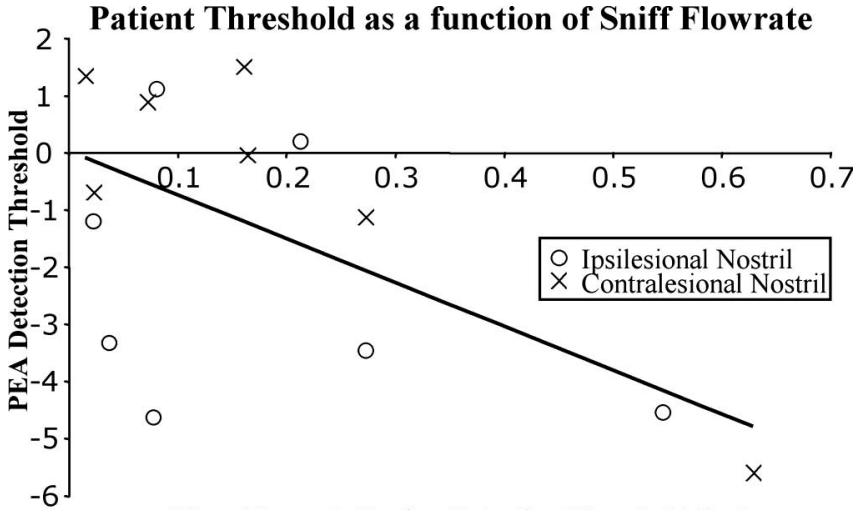

Mean Flowrate During Detection Threshold Task

Figure 4. Patient PEA thresholds as a function of mean sniff flow rate. Each point represents one nostril. A least-squares regression line is drawn in black. Patients who took larger sniffs had more negative (better) detection thresholds.

tralesional threshold $\left(r=0.22 ; F_{(1,5)}=0.24 ; p=0.64\right)$, indicating that the overall difference in sniff magnitude between the two sides was not sufficient to explain the laterality in threshold scores.

\section{Olfactomotor function}

Monorhinal testing

Perceived intensity during the task. Intensity ratings from the olfactomotor task are presented in Figure 5. A nested-design repeated-measures ANOVA on intensity ratings with factors of subject nested within group (patient, age-matched healthy, young healthy), side (ipsilateral matched, contralateral matched), and concentration (clean, low, medium, and high) revealed that, overall, intensity ratings were dependent on odorant concentration $\left(F_{(3,1245)}=320.87 ; p<0.0001\right)$. Critically, considering our intention to compare sniffing of low- and highintensity odorants, patients, age-matched controls, and young controls all gave significantly higher intensity ratings to the highcompared with low-concentration odorants $(p<0.0001$ in all cases).

Concentration-dependent sniffing. The mean sniffs generated for low- and high-concentration odorants during the monorhinal olfactomotor task are presented in Figure 6, A-F. Sniff volume was calculated for each trial (Fig. 7). A nested-design repeated-measures ANOVA with factors of subject nested within group (patient, age-matched healthy, young healthy), side (ipsilateral matched, contralateral matched), and concentration (low, high) revealed significant main effects of group $\left(F_{(2,919)}=114.08\right.$; $p<0.0001)$ and concentration $\left(F_{(1,919)}=6.5 ; p<0.011\right)$, and a significant interaction between group and side $\left(F_{(2,919)}=7.48\right.$; $p<0.0006)$.

Planned contrasts regarding absolute sniffing vigor regardless of odorant concentration revealed that patients took a significantly smaller sniff than both age-matched controls (mean patients, $24.39 \pm 1.14$; mean age-matched control, $46.24 \pm 1.27$; $\left.F_{(1,919)}=1447.26 ; p<0.0001\right)$ and young controls (mean, $\left.43.41 \pm 1.52 ; F_{(1,919)}=741.66 ; p<0.0001\right)$. Repeating this analysis using the measures of mean sniff velocity and maximum sniff velocity revealed a similar picture. However, in the analysis using sniff duration, the main effects of concentration and side were significant $\left(F_{(1,921)}=7.74 ; p<0.0082 ; F_{(1,921)}=6.65 ; p<0.01\right)$, but there was no effect of group $\left(F_{(2,921)}=1.83 ; p=0.16\right)$. In other words, in comparison with control subjects, patients' sniffs were less vigorous but equal in duration. 


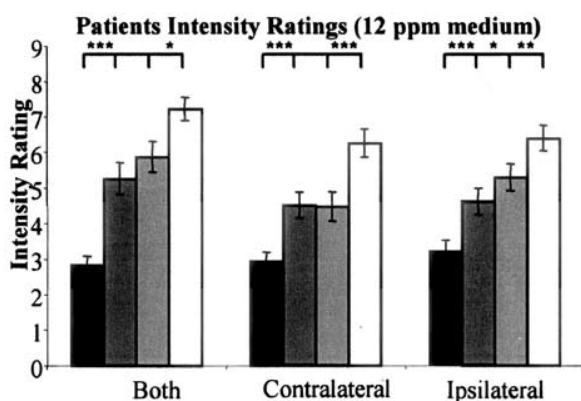

Age-matched Controls Intensity Ratings

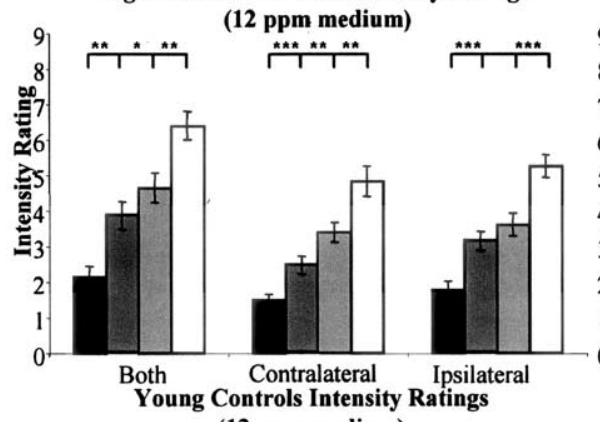

(12 ppm medium)

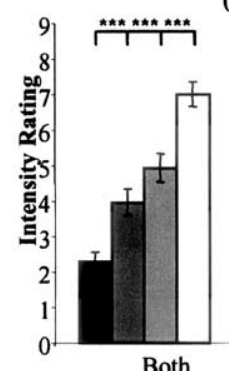

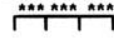

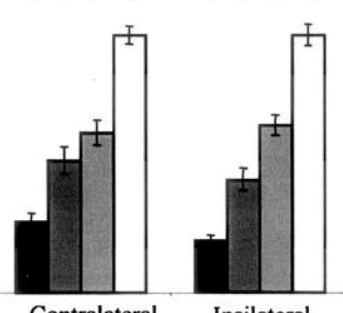

Ipsilatera

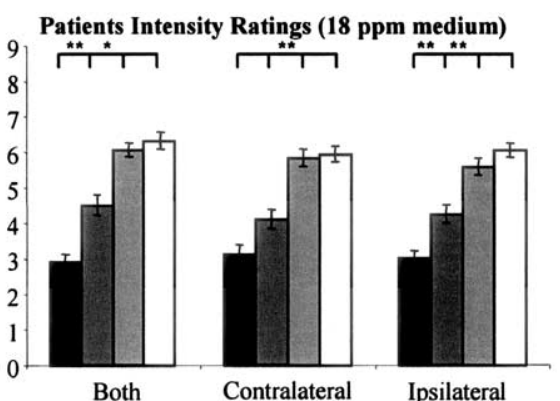

Age-Matched Controls Intensity Ratings (18 ppm medium)

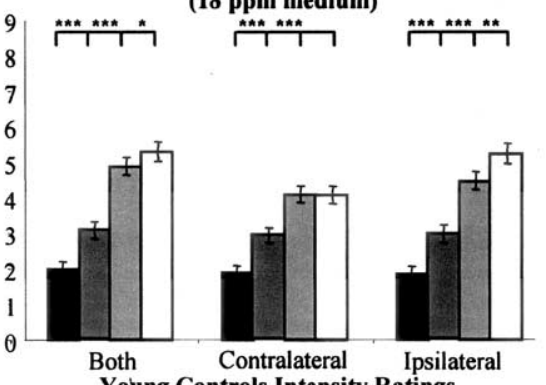

Young Controls Intensity Ratings (18 ppm medium)

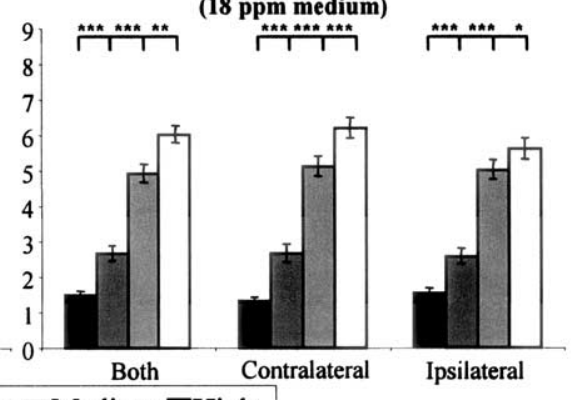

Medium $\square$ High

Figure 5. Intensity ratings during the olfactomotor task. Subjects rated the intensity of propionic acid on a scale from 1 (no odor) to 9 (very strong). Each bar represents the average of 28 sniffs ( 4 sniffs per condition for 7 subjects). Error bars indicate SE. ${ }^{*} p<0.05,{ }^{* *} p<0.01,{ }^{* * *} p<0.0001$, paired $t$ test. Note that patients showed a reduced ability to distinguish between neighboring concentrations relative to controls.

Planned contrasts regarding concentration-dependent sniff modulation revealed that young controls took a larger volume sniff for low-concentration odors than for high-concentration odors, although this effect was significant only in the ipsilesionalmatched nostril (ipsilesional, mean low, $40.86 \pm 3.51$; mean high, $33.46 \pm 2.66 ; F_{(1,919)}=10.38 ; p<0.0013$; contralesional, mean low, $44.38 \pm 2.97$; mean high, $41.39 \pm 3.10 ; F_{(1,919)}=1.69$; $p=0.19)$. In contrast, the patients and age-matched controls did not modulate the volume of their sniff in accordance with odorant concentration ( $p>0.49$ in all cases). Repeating the analysis with the measures of mean sniff velocity, maximum sniff velocity, and sniff duration, revealed similar results.

\section{Birhinal testing}

Monorhinal testing revealed that concentration-dependent sniff modulation was weak in young controls and absent in both agematched controls and patients. The weakness of the effect in the young subjects may be attributed to the small sample. Conversely, concentration-dependent sniff modulation has not been tested monorhinally in previous studies, and this phenomenon may be weaker or even absent when using only one nostril. To address this, we tested all subjects birhinally.

Perceived intensity during the task. Intensity ratings from the birhinal olfactomotor task are presented in Figure 5. A nested-design repeatedmeasures ANOVA on intensity ratings with factors of subject nested within group (patient, age-matched healthy, young healthy), side (ipsilateral matched, contralateral matched), and concentration (clean, low, medium, and high) revealed that intensity ratings varied with concentration $\left(F_{(3,560)}=42.92 ; p<0.0001\right)$.

Concentration-dependent sniffing. The mean sniffs generated for low- and highconcentration odorants during the birhinal olfactomotor task are presented in Figure 6, G-I. Sniff volume was calculated for each trial (Fig. 7). A nested-design repeated-measures ANOVA on sniff volume with factors of subject nested within group (patient, age-matched healthy, young healthy) and concentration (clean, low, medium, and high) revealed significant main effects of group $\left(F_{(2,441)}=\right.$ 124.99; $p<0.0001)$ and concentration $\left(F_{(1,441)}=11.66 ; p<0.0007\right)$.

Planned contrasts regarding absolute sniffing vigor regardless of odorant concentration revealed that patients took a significantly smaller sniff than both agematched controls (mean patients, $32.76 \pm$ 2.04; mean age-matched, $67.10 \pm 2.01$; $\left.F_{(1,441)}=60.32 ; p<0.0001\right)$ and young controls (mean young, $58.08 \pm 3.53$; $\left.F_{(1,441)}=32.90 ; p<0.0001\right)$. Similar to the monorhinal results, for sniff duration, the main effects of concentration and group were not significant $\left(F_{(1,454)}=1.82, p<\right.$ $0.18 ; F_{(2,454)}=2.76, p=0.064$, respectively). Thus, even when using both nostrils, the patients' sniffs were less vigorous than control subjects' sniffs, but equal in

duration.

Planned comparisons regarding concentration-dependent sniff modulation revealed that young controls varied their sniff volume with concentration (mean young low, $60.65 \pm 5.00$; mean young high, $\left.55.54 \pm 5.00 ; F_{(1,441)}=4.02 ; p<0.046\right)$. In contrast, there was only a moderate trend in age-matched controls, and a weaker trend in patients toward concentration modulation of sniff volume (mean age-matched low, $69.50 \pm 2.95$; mean age-matched high, $64.61 \pm 2.70 ; F_{(1,441)}=3.69 ; p=0.055$; mean patient low, $34.83 \pm 3.03$; mean patient high, $30.72 \pm 2.74$; $\left.F_{(1,441)}=2.60 ; p=0.11\right)$. Repeating the analysis with the measures of mean sniff velocity, maximum sniff velocity, and sniff duration, revealed similar results.

\section{Maximal sniff generation}

The olfactomotor testing revealed that patients generated sniffs that were significantly less vigorous than sniffs of age-matched control subjects, regardless of odorant concentration. This raised the possibility that patients were simply unable, in terms of respiratory capacities, to generate and control the necessary sniff. To address this possibility, we asked patients to generate their maximal nasal inspiration. This maximal nasal inspiration was significantly greater in volume than the sniffs used during the tasks by 
age-matched (maximum patient volume, $77.07 \pm 23.47$; mean age-matched volume, $23.66 \pm 2.58 ; t_{(17)}=3.36 ; p<$ 0.0037 ) and young (mean young volume, $\left.18.11 \pm 3.59 ; t_{(17)}=3.70 ; p<0.0018\right)$ subjects, suggesting that the reduced sniffing during the olfactory tasks was not a reflection of respiratory limitations.

\section{Effects of lesion size and location}

To test for a relationship between lesion size and location and the olfactory impairments, each patient was assigned a composite score based on equally weighted UPSIT and detection threshold scores. Lesion size (volume) did not correlate with the overall olfactory score $(r=0.37 ; p=$ 0.42 ).

Next, we gave each patient an olfactomotor score that reflected concentration modulation of the sniff. We took the average velocity of the patient's low concentration sniffs and subtracted the average velocity of the patient's high concentration sniffs. There was a trend toward a correlation, whereby a larger lesion predicted better concentration-dependent modulation $(r=0.52 ; p=0.067)$. Note that the potential strength of these comparisons was limited by the sample size.

\section{Discussion}

A contralesional olfactory impairment in patients

Patients with unilateral cerebellar lesions were impaired at olfactory identification.

These impairments were most pronounced when the odorant was restricted to the contralesional nostril. In contrast, patients initially appeared unimpaired at olfactory detection in both nostrils. This accuracy and symmetry in olfactory detection was paradoxical in view of the inaccuracy and asymmetry in olfactory identification. We hypothesized that patients may have modified their sniffing strategies when restricted to sniffing with the contralesional (impaired) nostril, and that such compensation was sufficient to generate equal performance across nostrils. To address this possibility, we obtained detection thresholds under conditions in which the patients were required to sniff in an equal manner with both nostrils. This manipulation revealed an impairment in the patients' detection threshold. Critically, patients also showed significantly greater impairment in the contralesional nostril. One might argue that the increased demands of the fixed-sniff task posed greater difficulty for the patients compared with the control subjects, and that this increased difficulty underlies their poorer performance. Such an argument, however, fails to account for the pronounced asymmetry in patient fixed-sniff detection threshold.

\section{Sensory rather than cognitive sources of impairment}

We considered whether the impairment in olfactory identification was of cognitive rather than olfactory origin. This hypothesis seems unlikely because of the following factors. First, the elevated detection thresholds in the fixed-sniff condition reflect an olfactory impairment in a task with minimal cognitive demands. Sec- ond, standardized tests (WAIS-III, BNT, WMS) suggested that the patients were mostly unimpaired in the cognitive domains essential for performing an identification task. Third, the asymmetry in performance between the two nostrils further supports a genuinely olfactory rather than cognitive impairment, given that generalized cognitive impairments would likely affect performance with either nostril. Fourth, in contrast to vision, olfaction maintains an ipsilateral path from periphery to cortex (Price, 1990). Concordantly, whereas unilateral cerebellar lesions lead to ipsilesional impairments in motor and visual abilities (Snider and Stowell, 1944; Suzuki and Keller, 1988; Bjaalie and Brodal, 1989), here such lesions led to a contralesional olfactory impairment. Thus, the reversed laterality of the impairment-to-lesion relationship mirrored the reversed laterality of the early sensory projections. This link between sensory organization and observed impairment further links the impairment to sensory rather than cognitive capacities. The only evidence that suggested that cognitive components might have contributed to the impairment in olfactory identification was the patients' poor performance on the auditory identification test. Critically, however, this impairment was not lateralized, suggesting that the mechanisms behind it are distinct from the strongly lateralized mechanisms underlying the impairment in olfactory identification.

Possible paths from nose to cerebellum

Despite evidence for a cerebellar role in olfaction seen in imaging, human lesions, and genetic mouse models (Feron and Baudoin, 1992, 1993; Baudoin et al., 1994; Deiss and Baudoin, 1997), an 

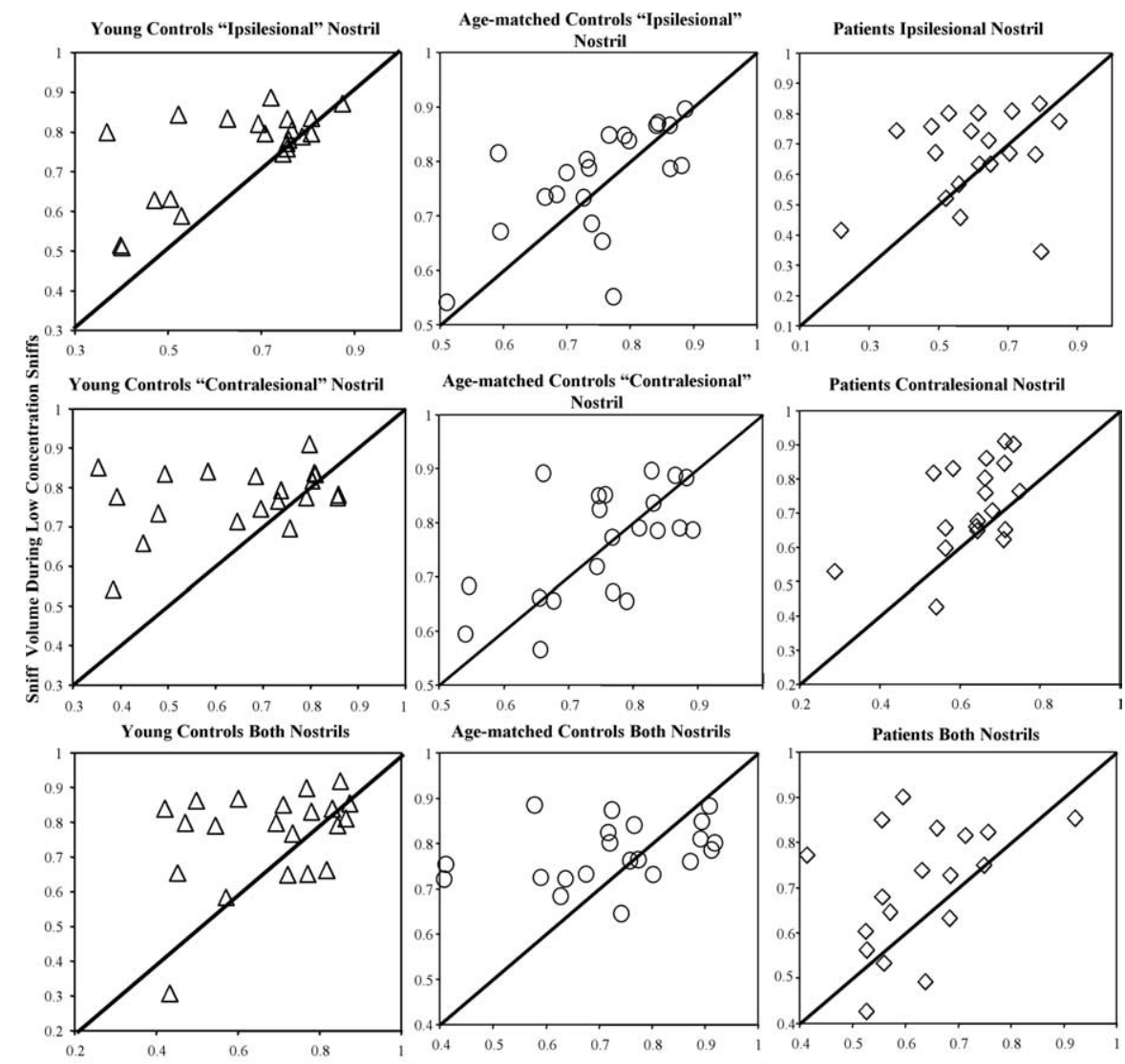

Sniff Volume During High Concentration Sniffs

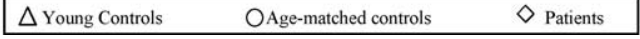

Figure 7. Effect of odor concentration on sniff volume. Each symbol represents the average of data from one block ( 3 blocks per subject). Data were normalized on an individual basis, using the maximum value for sniff volume. Concentration-dependent modulation of the sniff is reflected in points accumulated on one side of the line, and concentration-independent sniffing is reflected in points distributed around the line. Note that the points representing young controls accumulated mostly above the line, whereas points representing age-matched controls and patients were distributed around the line.

olfactocerebellar pathway has yet to be described. Ikai et al. (1992, 1994) found that single neurons in the rat's ventral tegmental area send collaterals to both primary olfactory (piriform) cortex and both hemispheres of the cerebellum. However, no direct evidence has shown that this pathway mediates olfactory information. The fifth cranial nerve may also relay odor information to the cerebellum, given that it projects to the cerebellum (Kiernan, 1998), and its nerve endings are responsive to high concentrations of most odorants (Doty et al., 1978; Doty, 1995). The current results suggest an initial set of constraints in characterizing the olfactocerebellar pathway, indicating that, from a functional perspective, the projection from nose to cerebellum is primarily contralateral.

\section{Cerebellar involvement in the olfactomotor system}

The cerebellum is functionally well situated to generate and/or modulate olfactomotor responses. Consistent with its role in respiration, the cerebellum may interact with either spinal inspiratory facilitatory reflexes or supraspinal inspiratory inhibitory reflexes to modify the diaphragm and intercostal muscles that control the sniff (Decima and von Euler, 1969). Depression of cerebellar function in the cat by ischemia, topical administration of procaine, or ablation, results in an augmentation of inspiratory activity (Glasser et al., 1966). In addition, electrical stimulation of the anterior lobe of the cerebellum inhibits the inspiratory dis- charge driven by the medullary respiratory mechanism (Moruzzi, 1940; Decima and von Euler, 1969). Together, these results suggest that the cerebellum provides a tonic, primarily inhibitory, influence on inspiratory mechanisms of the lower brainstem. Furthermore, patients with cerebellar lesions often have weakness in the muscles of the diaphragm and show respiratory ataxia (Mavlov and Chavdarov, 1968; Mier-Jedrzejowicz and Green, 1988).

Cerebellar involvement in the olfactomotor system can be viewed within two conceptual frameworks. One stresses the role of the cerebellum in general motor control (Holmes, 1939; Thach et al., 1992; Ito, 1993). Within this framework, cerebellar lesions are predicted to hamper overall olfactomotor ability or, put simply, the ability to sniff. A second framework stresses the role of the cerebellum in sensorimotor control specifically to optimize sensory processing (Bower, 1997a,b). For example, cerebellar involvement in the optokinetic reflex helps stabilize visual signals during head and body movement (Robinson, 1976; Lisberger and Sejnowski, 1992). In light of the anatomical uniformity of cerebellar circuits (Palay and Chan-Palay, 1974), we hypothesized that the cerebellum may play a similar role in olfaction. In this view, the cerebellum might use sensory information from the olfactory system to modulate movements of the diaphragm and intercostal muscles to create the olfactomotor response, a sniff, that in turn would optimize sensory processing. Our results are consistent with either framework.

\section{Reduced absolute sniffing ability in patients}

Consistent with a general cerebellar role in motor control, patients failed to generate vigorous sniffs. Their sniffs were small in flow rate and volume, but not duration, suggesting that it was not a lack of motivation that inhibited their sniffing behavior. In contrast, the maximal sniffs generated by the patients were more vigorous than the sniffs they generated during the olfactory tasks, or the sniffs generated by healthy subjects during the tasks (Fig. 8 ). This suggests that, although patients were not unable to sniff, they did not generate sufficient sniffs within the olfactory context. Olfactory performance was dependent on sniffing; patients who took more vigorous sniffs had better olfactory detection thresholds (Fig. 4). Thus, we can link the cerebellar lesions to reduced sniffing and link reduced sniffing to reduced olfactory performance, but, at present, we cannot claim causality in this link. That is, we do not know whether the patients sniffed less because they had poorer olfaction, or whether they had poorer olfaction because they sniffed less, as suggested in Parkinson's disease (Sobel et al., 2001).

\section{Reduced concentration-dependent sniff modulation in patients and age-matched controls}

Consistent with a cerebellar role in sensorimotor control to optimize sensory processing, the patients did not modulate sniff 


\section{Sniff Volume}

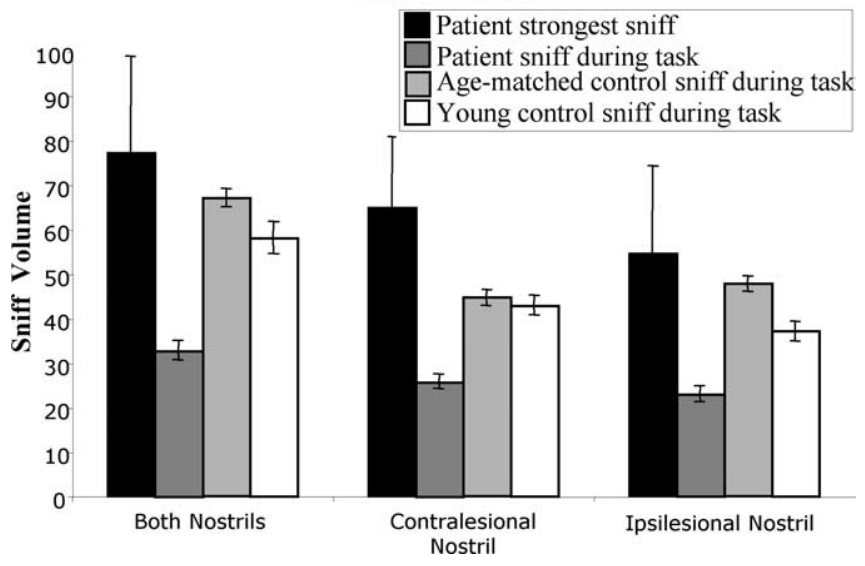

Figure 8. Average sniff volume during olfactomotor performance. The bar representing the patient's strongest sniff was the average of seven sniffs (1 per patient). All other bars were the average of 168 sniffs. Error bars represent SE. Note that, although both control groups took significantly larger sniffs than patients during the task, when patients were asked to take their strongest sniff, they were capable of taking larger sniffs than those taken by control participants during the task.

volume in accordance with odorant concentration. However, age-matched healthy subjects also did not reliably modulate sniff volume in accordance with odorant concentration, although there was a trend in that direction $(p<0.055)$. Only the young subjects generated the expected inverse concentrationdependent sniff profile. Assuming the function of concentrationdependent sniffing is to compute veridical odorant concentrations (i.e., concentration constancy), we would predict that both patients and age-matched controls would report more similar perceived intensities across different concentrations than would young controls. This is precisely what we observed. That said, the small differences between patients and age-matched controls in this respect render any current assessment of cerebellar involvement in this mechanism inconclusive. Furthermore, patients sniffed in a way that partly compensated for their olfactory impairment, suggesting that some aspects of the olfactomotor system were intact, and that the neural substrates of the olfactomotor system may be, at least in part, of extracerebellar origin (Vanderwolf, 2001).

\section{Conclusion}

Patients with unilateral focal cerebellar lesions offer a unique opportunity to assess functional laterality, with each patient serving as their own control. Unfortunately, identifying such patients is difficult, with our sample size limited to seven participants even after several years of recruitment. To overcome the limited statistical power of this sample, we retested the patients multiple times and throughout this paper have opted to present scatter graphs to convey a full and accurate picture of the data. Despite the drawbacks of a limited subject pool, lesion patient data provide a vital insight toward establishing a cerebellar role in olfaction. That the cerebellum was "activated" in imaging studies of olfaction alone does not prove a functional role for the cerebellum in the olfactory process. Such a functional role can be deduced only by combining multiple sources of information. In this respect, the current results dovetail nicely with previous results implicating the cerebellum in olfactory identification (Abele et al., 2003; Connelly et al., 2003) and further highlight the genuine olfactory origin of this impairment. Finally, the lateralization pattern of these results provides an initial set of constraints toward mapping the functional path from nose to cerebellum and allows us to conclude that this primarily contralateral path plays a functional role in olfaction.

\section{References}

Abele M, Riet A, Hummel T, Klockgether T, Wullner U (2003) Olfactory dysfunction in cerebellar ataxia and multiple system atrophy. J Neurol 250:1453-1455.

Baudoin C, Feron C, Deiss V (1994) Olfactory Deficits induced by the murine staggerer mutation: behavioural data. Adv Biosci 93:413-419.

Bjaalie JG, Brodal P (1989) Visual pathways to the cerebellum: segregation in the pontine nuclei of terminal fields from different visual cortical areas in the cat. Neuroscience 29:95-107.

Bower JM (1997a) Control of sensory data acquisition. Int Rev Neurobiol 41:489-513.

Bower JM (1997b) Is the cerebellum sensory for motor's sake, or motor for sensory's sake: the view from the whiskers of a rat? Prog Brain Res 114:463-496.

Buckner RL, Petersen SE, Ojemann JG, Miezin FM, Squire LR, Raichle ME (1995) Functional anatomical studies of explicit and implicit memory retrieval tasks. J Neurosci 15:12-29.

Cain WS, Johnson Jr F (1978) Lability of odor pleasantness: influence of mere exposure. Perception 7:459-465.

Cerf-Ducastel B, Murphy C (2001) fMRI activation in response to odorants orally delivered in aqueous solutions. Chem Senses 26:625-637.

Connelly T, Farmer JM, Lynch DR, Doty RL (2003) Olfactory dysfunction in degenerative ataxias. J Neurol Neurosurg Psychiatry 74:1435-1437.

Decima EE, von Euler C (1969) Intercostal and cerebellar influences on efferent phrenic activity in the decerebrate cat. Acta Physiol Scand 76:148-158.

Deiss V, Baudoin C (1997) Hyposmia for butanol and vanillin in mutant staggerer male mice. Physiol Behav 61:209-213.

Desmond JE, Fiez JA (1998) Neuroimaging studies of the cerebellum: language, learning, and memory. Trends Cogn Sci 2:355-362.

Doty RL (1992) Diagnostic tests and assessment. J Head Trauma Rehabil 7:45-64.

Doty RL (1995) Intranasal trigeminal chemoreception: anatomy, physiology, and psychophysics. In: Handbook of olfaction and gustation (Doty RL, ed), pp 821-834. New York: Marcel Dekker.

Doty RL, Brugger WE, Jurs PC, Orndorff MA, Snyder PJ, Lowry LD (1978) Intranasal trigeminal stimulation from odorous volatiles: psychometric responses from anosmic and normal humans. Physiol Behav 20:175-185.

Doty RL, Shaman P, Dann M (1984) Development of the University of Pennsylvania Smell Identification Test: a standardized microencapsulated test of olfactory function. Physiol Behav 32:489-502.

Ferdon S, Murphy C (2003) The cerebellum and olfaction in the aging brain: a functional magnetic resonance imaging study. NeuroImage 20:12-21.

Feron C, Baudoin C (1992) Reaction of staggerer and nonmutant mice to female urine and vaginal secretion odors. Behav Proc 27:165-170.

Feron C, Baudoin C (1993) Sexual experience and preferences for odors of estrous females in staggerer mutant male mice. Behav Neural Biol 60:280-281.

Frank RA, Dulay MF, Gesteland RC (2003) Assessment of the Sniff Magnitude Test as a clinical test of olfactory function. Physiol Behav 78:195-204.

Glasser RL, Tippett JW, Davidian Jr VA (1966) Cerebellar activity, apneustic breathing, and the neural control of respiration. Nature 209:810-812.

Good KP, Martzke JS, Daoud MA, Kopala LC (2003) Unirhinal norms for the University of Pennsylvania Smell Identification Test. Clin Neuropsychol 17:226-234.

Holmes G (1939) The cerebellum of a man. Brain 40:461-535.

Hore J, Wild B, Diener HC (1991) Cerebellar dysmetria at the elbow, wrist, and fingers. J Neurophysiol 65:563-571.

Ikai Y, Takada M, Shinonaga Y, Mizuno N (1992) Dopaminergic and nondopaminergic neurons in the ventral tegmental area of the rat project, respectively, to the cerebellar cortex and deep cerebellar nuclei. Neuroscience 51:719-728.

Ikai Y, Takada M, Mizuno N (1994) Single neurons in the ventral tegmental area that project to both the cerebral and cerebellar cortical areas by way of axon collaterals. Neuroscience 61:925-934.

Ito M (1993) Movement and thought: identical control mechanisms by the cerebellum. Trends Neurosci 16:448-450. 
Johnson BN, Mainland JD, Sobel N (2003) Rapid olfactory processing implicates subcortical control of an olfactomotor system. J Neurophysiol 90:1084-1094.

Kendal-Reed M, Walker JC, Morgan WT, LaMacchio M, Lutz RW (1998) Human responses to propionic acid. I. Quantification of within- and between-participant variation in perception by normosmics and anosmics. Chem Senses 23:71-82.

Kendal-Reed M, Walker JC, Morgan WT (2001) Investigating sources of response variability and neural mediation in human nasal irritation. Indoor Air 11:185-191.

Kiernan JA (1998) The human nervous system, Ed 7. Philadelphia: Lippincott Williams and Wilkins.

Laing DG (1983) Natural sniffing gives optimum odour perception for humans. Perception 12:99-117.

Linschoten MR, Harvey Jr LO, Eller PM, Jafek BW (2001) Fast and accurate measurement of taste and smell thresholds using a maximum-likelihood adaptive staircase procedure. Percept Psychophys 63:1330-1347.

Lisberger SG, Sejnowski TJ (1992) Motor learning in a recurrent network model based on the vestibulo-ocular reflex. Nature 360:159-161.

Marcell MM, Borella D, Greene M, Kerr E, Rogers S (2000) Confrontation naming of environmental sounds. J Clin Exp Neuropsychol 22:830-864.

Mavlov L, Chavdarov D (1968) Investigations of respiratory ataxia in cerebellar lesions. Dokl Bulg Acad Nauk 21:1237-1238.

Mier-Jedrzejowicz A, Green M (1988) Respiratory muscle weakness associated with cerebellar atrophy. Am Rev Respir Dis 137:673-677.

Moruzzi G (1940) Paleocerebellar inhibition of vasomotor and respiratory carotid sinus reflexes. J Neurophysiol 3:20-32.

Moscovitch C, Kapur S, Kohler S, Houle S (1995) Distinct neural correlates of visual long-term memory for spatial location and object identity: a positron emission tomography study in humans. Proc Natl Acad Sci USA 92:3721-3725.

Palay SL, Chan-Palay V (1974) Cerebellar cortex: cytology and organization. Berlin: Springer.

Price JL (1990) Olfactory system. In: The human nervous system (Paxinos G, ed), pp 979-1001. San Diego: Academic.

Qureshy A, Kawashima R, Imran MB, Sugiura M, Goto R, Okada K, Inoue K, Itoh M, Schormann T, Zilles K, Fukuda H (2000) Functional mapping of human brain in olfactory processing: a PET study. J Neurophysiol 84:1656-1666.
Robinson DA (1976) Adaptive gain control of vestibuloocular reflex by the cerebellum. J Neurophysiol 39:954-969.

Savic I, Gulyas B, Larsson M, Roland P (2000) Olfactory functions are mediated by parallel and hierarchical processing. Neuron 26:735-745.

Small DM, Jones-Gotman M, Zatorre RJ, Petrides M, Evans AC (1997) A role for the right anterior temporal lobe in taste quality recognition. J Neurosci 17:5136-5142.

Snider RS, Stowell A (1944) Receiving areas of the tactile, auditory and visual systems in the cerebellum. J Neurophysiol 7:331-357.

Sobel N, Prabhakaran V, Hartley CA, Desmond JE, Zhao Z, Glover GH, Gabrieli JD, Sullivan EV (1998) Odorant-induced and sniff-induced activation in the cerebellum of the human. J Neurosci 18:8990-9001.

Sobel N, Khan RM, Hartley CA, Sullivan EV, Gabrieli JD (2000) Sniffing longer rather than stronger to maintain olfactory detection threshold. Chem Senses 25:1-8.

Sobel N, Thomason ME, Stappen I, Tanner CM, Tetrud JW, Bower JM, Sullivan EV, Gabrieli JD (2001) An impairment in sniffing contributes to the olfactory impairment in Parkinson's disease. Proc Natl Acad Sci USA 98:4154-4159.

Suzuki DA, Keller EL (1988) The role of the posterior vermis of monkey cerebellum in smooth-pursuit eye movement control. I. Eye and head movement-related activity. J Neurophysiol 59:1-18.

Thach WT, Goodkin HP, Keating JG (1992) The cerebellum and the adaptive coordination of movement. Annu Rev Neurosci 15:403-442.

Tsukatani T, Miwa T, Furukawa M, Costanzo RM (2003) Detection thresholds for phenyl ethyl alcohol using serial dilutions in different solvents Chem Senses 28:25-32.

Vanderwolf CH (2001) The hippocampus as an olfacto-motor mechanism: were the classical anatomists right after all? Behav Brain Res 127:25-47.

Walker JC, Kendal-Reed M, Hall SB, Morgan WT, Polyakov VV, Lutz RW (2001) Human responses to propionic acid. II. Quantification of breathing responses and their relationship to perception. Chem Senses 26:351-358.

Yousem DM, Williams SC, Howard RO, Andrew C, Simmons A, Allin M, Geckle RJ, Suskind D, Bullmore ET, Brammer MJ, Doty RL (1997) Functional MR imaging during odor stimulation: preliminary data. Radiology 204:833-838.

Zatorre RJ, Jones-Gotman M, Rouby C (2000) Neural mechanisms involved in odor pleasantness and intensity judgments. NeuroReport 11: 2711-2716. 